\title{
Automated grading of homework assignments and tests in introductory and intermediate statistics courses using active server pages
}

\author{
DAVID W. STOCKBURGER \\ Southwest Missouri State University, Springfield, Missouri
}

\begin{abstract}
Active server pages permit a software developer to customize the Web experience for users by inserting server-side script and database access into Web pages. This paper describes applications of these techniques and provides a primer on the use of these methods. Applications include a system that generates and grades individualized homework assignments and tests for statistics students. The student accesses the system as a Web page, prints out the assignment, does the assignment, and enters the answers on the Web page. The server, running on NT Server 4.0, grades the assignment, updates the grade book (on a database), and returns the answer key to the student.
\end{abstract}

When Microsoft introduced active server pages (ASP) in 1996-1997, it became possible to modify and generate hypertext markup language (HTML) code from programs located on a Web server. By combining information from the user in response to forms on a Web page and information stored in databases, the Web experience can be customized for each user. ASP are similar to CGI (Common Gateway Interface) but may be programmed in a subset of either Visual Basic (VBScript) or Java (Jscript). The first section of this paper discusses an application of ASP to generate and score individualized tests and homework assignments for statistics students. After that, a primer presents the two key concepts in using ASP in Web development. Technical issues in the use of the technology are then followed by a discussion of additional applications.

\section{INDIVIDUALIZED TESTS AND HOMEWORK ASSIGNMENTS FOR STATISTICS STUDENTS}

Grading and recording tests and homework assignments are probably the least-rewarding tasks of teaching statistics. Using a computer to automate these tasks can save the instructor hours of nonproductive time. In addition, students receive individualized homework assignments and tests, along with immediate feedback as to the correctness of their answers. Examples of assignments and tests are easily given to students with this system. This pre-

Correspondence concerning this article should be addressed to D. W. Stockburger, Psychology Department, Southwest Missouri State University, Springfield, MO 65804 (e-mail: dws148f@mail.smsu.edu). sentation mode is ideal for a distance learning and Web course environment.

An automatic grading system, expanding an earlier version (Stockburger, 1996), can be previewed [at http:// www.psychstat.smsu.edu]. From the home page, select one of the course pages (e.g., PSY 200-Introductory Statistics) and use the default values to log on as "Example Student." The various exercises and tests can then be viewed from this page. Additional examples of tests and assignments can be viewed by clicking the "Example Tests and Assignments" button on any course page.

Starting with the course page (see Figure 1), students $\log$ on to their gradebook page by entering an account code and password, given on the 1 st day of class. The gradebook page (Figure 2) is a customized Web page generated from database tables. The gradebook page presents the current status of the student, including which assignments have been submitted, the score received for each of the submitted assignments, the due dates for all assignments, and the maximum score received by any student thus far. The "Example Student" in the example would be replaced with the actual student's name. All assignments are available to the student at all times. The button to generate a given test, however, does not appear until after the day and time appearing on the gradebook, allowing considerable flexibility in determining when a given student takes a test.

Using this arrangement, an individualized assignment is generated for each student. By storing a random number seed as a value in the database, the same sequence of numbers may be generated on demand. The homework assignments and tests are presented as forms to the student, containing similar problems for all the students but with different numbers. If no grade is recorded for a given 


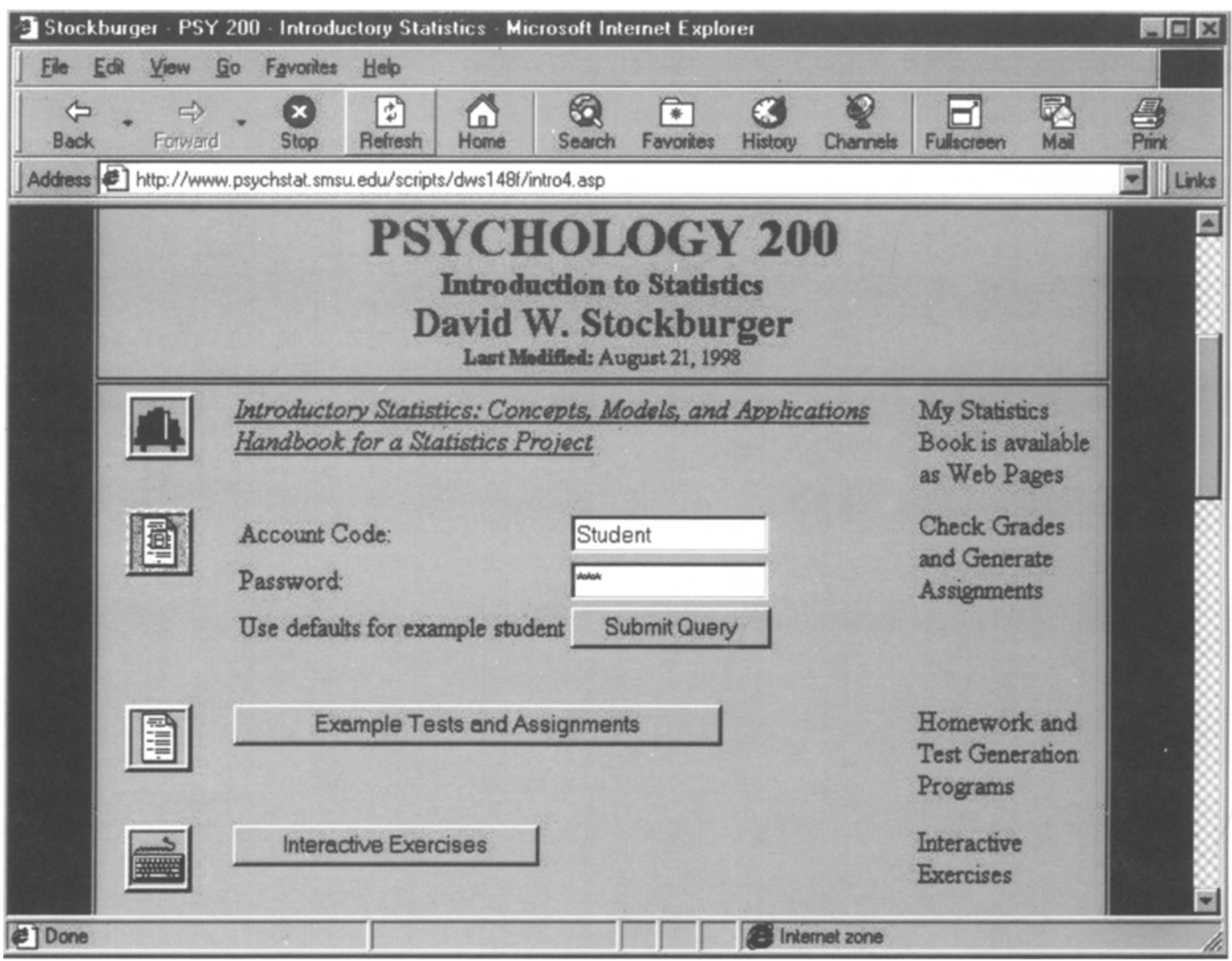

Figure 1. Course Web page.

assignment, the text boxes on the form are empty. An example assignment is presented in Figure 3.

When the student has completed the assignment, he or she regenerates the assignment as a Web page and fills in the form with the correct answers, clicking on the "Submit" button. The server grades the assignment, updates the database, and returns the answer key to the student as a filled-in form (see Figure 4). Additional information is presented to the student when an item is missed, including the student's response, the correct answer, and a pointer to the section of the on-line text dealing with the item. The student always receives at least one point after hitting the "Submit" button, so that the next time the student retrieves the assignment, the answer key will be presented.

Fifteen homework assignments and three tests have been completed for the introductory statistics course. The intermediate class has seven additional assignments and three tests available. The assignments range in difficulty from a simple review of algebra to using a cluster analysis algorithm to generate a dendogram. An algorithm to find the inverse of a matrix permits fairly complex multiple regression problems to be presented with this system.

This arrangement has provided considerable flexibility as a teaching tool. Students can submit assignments and tests at any time (early or late) and any place (home or laboratory), with no inconvenience to the instructor. Record keeping is minimized, and backups of the database are kept on several different computers. Students also have the ability to access their current scores on assignments and tests at any time. Student reaction has generally been favorable, after the initial shock of the extensive use of computers has abated.

The system works by taking the information provided by the student at log-on, comparing it with keys in a database, and if matches are found, retrieving additional information from the database for use in a personalized Web page. The additional information retrieved includes the student's full name and scores on all the tests and assignments. Critical skills to write code that performs these tasks include the creation of a database and retrieval of database records through the active server pages.

\section{ASP TUTORIAL}

Client-side scripts are programs passed to the user on the Web page. They have the advantage of accessing the server a single time, thus reducing the traffic on the Web and the load on the server. Not all browsers are equipped 


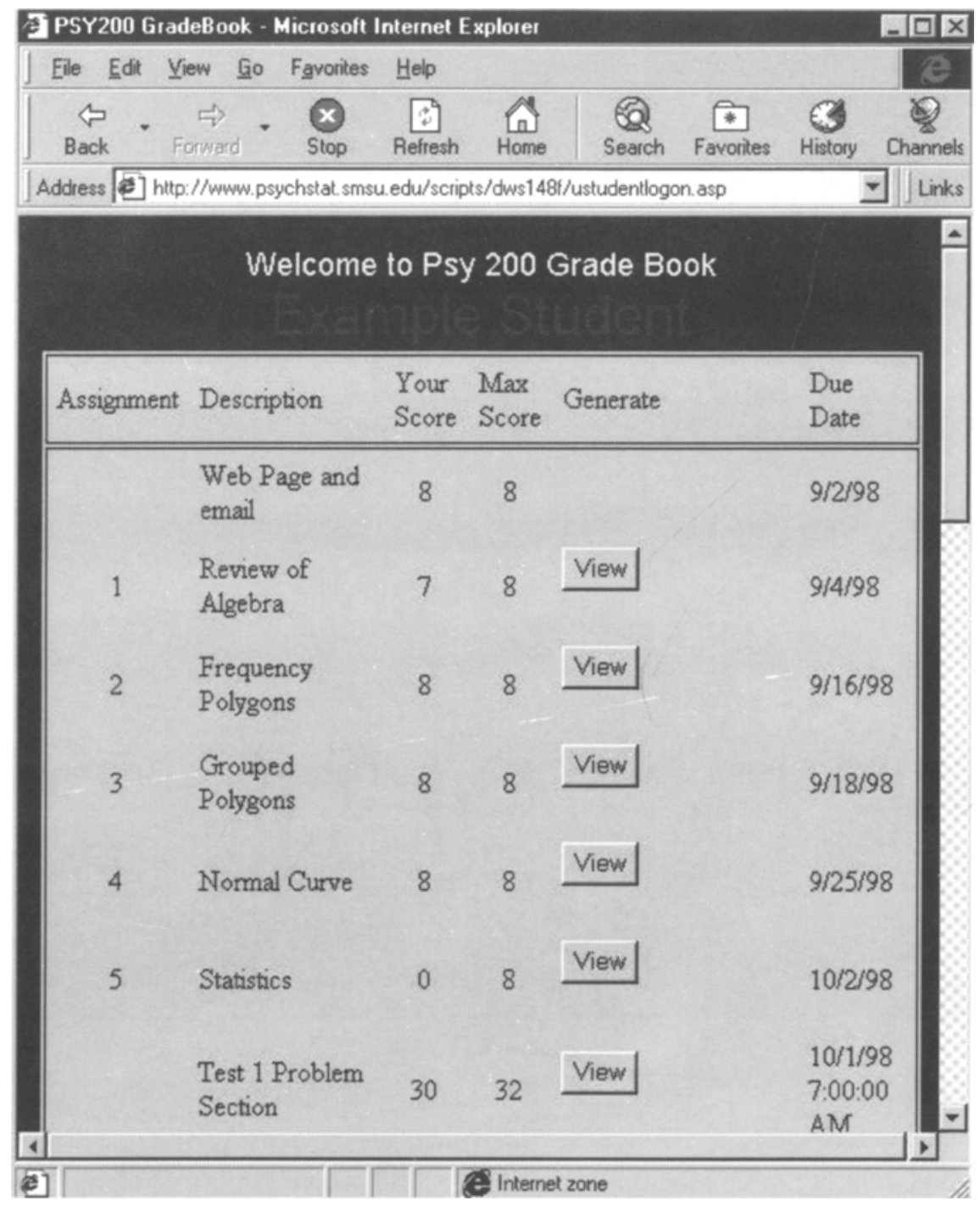

Figure 2. Example gradebook page.

to run scripts, and some scripts will only run on certain browsers. VBScript, for example, will not run on Netscape browsers. Because the code is passed to the user, it is open to user inspection.

Server-side scripts are programs that run on the server and require a transfer from the client to the server and back to the client every time a script is run. Each user places a load on the server, so that many users simultaneously running scripts can degrade the performance of the server. Server-side scripts are run in a protected and known environment (the server) and allow the programmer to read and write text and database files. Server-side scripts are not available for inspection by the user unless the system specifically allows the user to read the files. Server-side script includes all text between the " $<\%$ " and "\%>" markers on the ASP Web page. The listings for the example programs present server-side script with a gray background.

Even though it is not absolutely necessary, ASPs generally contain some server-side script. Although ASPs may be programmed with any ASCII editor, Microsoft has made the job easier by providing a software package called INTERDEV. This package exposes the properties and methods of the objects and was designed with database programming in mind. A source of information about ASP is Corning, Elfanbaum, and Melnick (1997).

The easiest way to obtain information from the user is through the use of forms (see Kenton, 1998). The information entered on the form by the user can be directly accessed by the client-side scripts or passed to server-side scripts. A combination of client- and server-side scripting can often provide an optimal environment for Web development.

\section{Client and Server-Side Scripts With Forms}

A simple program (Listing 1) demonstrates the use of both client- and server-side scripts. A Web page (Figure 5) requests that the user enter five numbers. After entering the numbers, the user can request that the program 


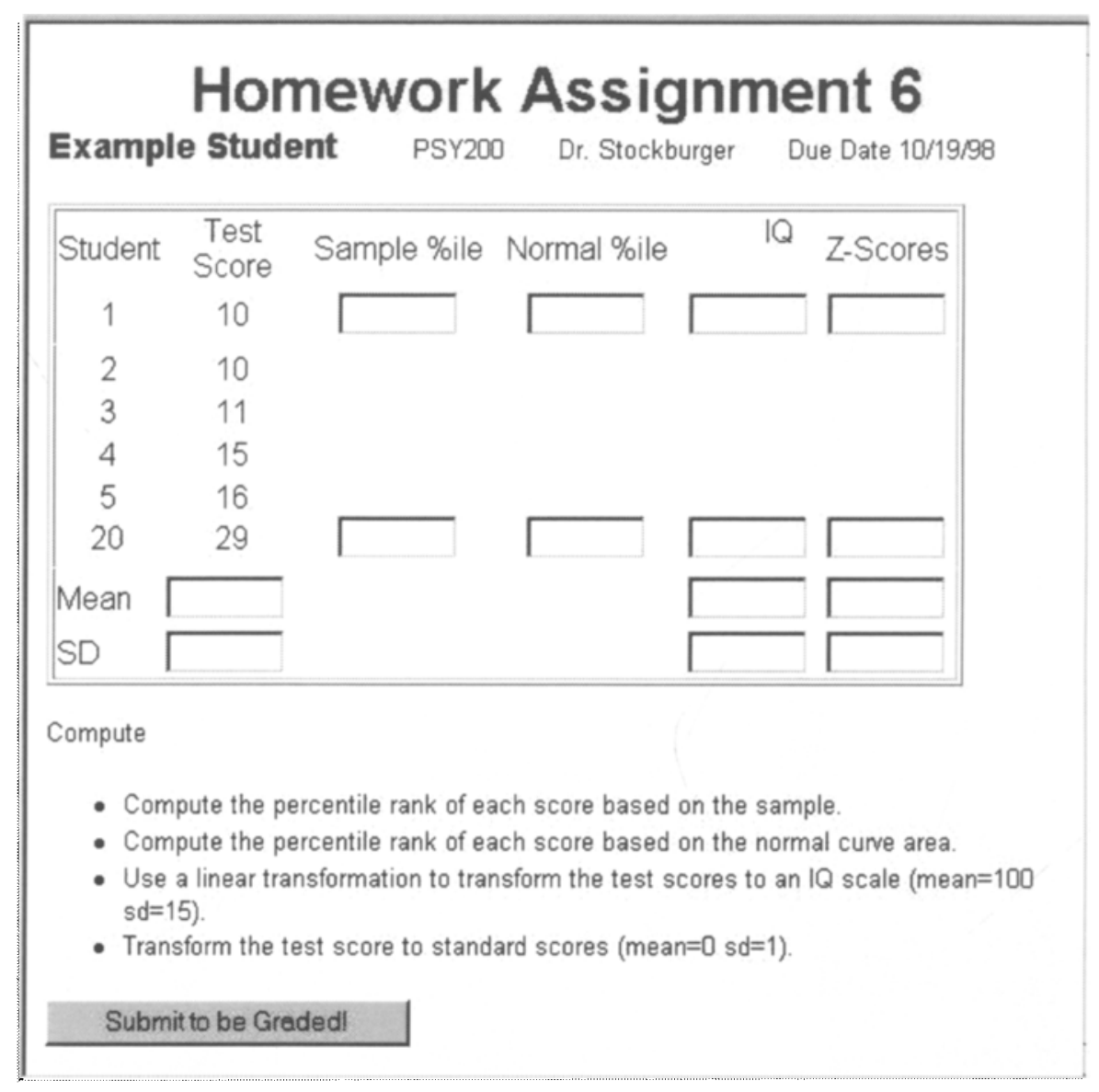

Figure 3. Example assignment.

calculate the mean and standard deviation, using a clientor server-side script. The client-side program uses Jscript, to be compatible with both Microsoft and Netscape browsers. The server-side script is written in VBScript and is highlighted in the program listing. In order to simplify the listing, data checks to ensure that user entries are numbers are not performed.

Setting up the program. The demonstration program may be entered directly, using any ASCII editor, or may be obtained from http://www.psychstat.smsu.edu/BRMIC/ BRMIC.zip as a zipped file. In either case, the file must be located in a directory accessible from an ASP-enabled Web server and the "Execute" option must be selected in the "Web Sharing" tab on the "Properties" of the program folder.

Running the program. The user runs and experiences ASP programs like any other Web page. Entering http:// www.psychstat.smsu.edu/scripts/BRMIC/BRMIC-1.asp will run the example page. The first time the page is called, the server finds no information on the form (the numbers have not yet been entered), so the data entries are set to zero and the mean and standard deviation boxes are empty. The program will be recalled and rerun when the user clicks on the "Server-Side" button, because the "action" property of the "form" object has been set to call itself "BRMIC-1 asp." If the user has entered numbers in the text boxes, the server will find the mean and standard deviation of the numbers and include them on the page when the page is returned to the client.

When the user clicks the "Client-Side" button, the page calls the "FindStats()" function on the client. This function immediately updates the text boxes on the page with the mean and standard deviation of the five numbers. It does so without action on the part of the server.

Program details. A few details of the script may be unfamiliar to the programmer who has some experience with BASIC or Java programming and will be discussed in the following section. The Request.form("name") command will be replaced with the user entry in the text box called "name". For example, the Request.form(" $x 1$ ") command will transfer the entry in the text box named " $x 1$ " to the current program. 


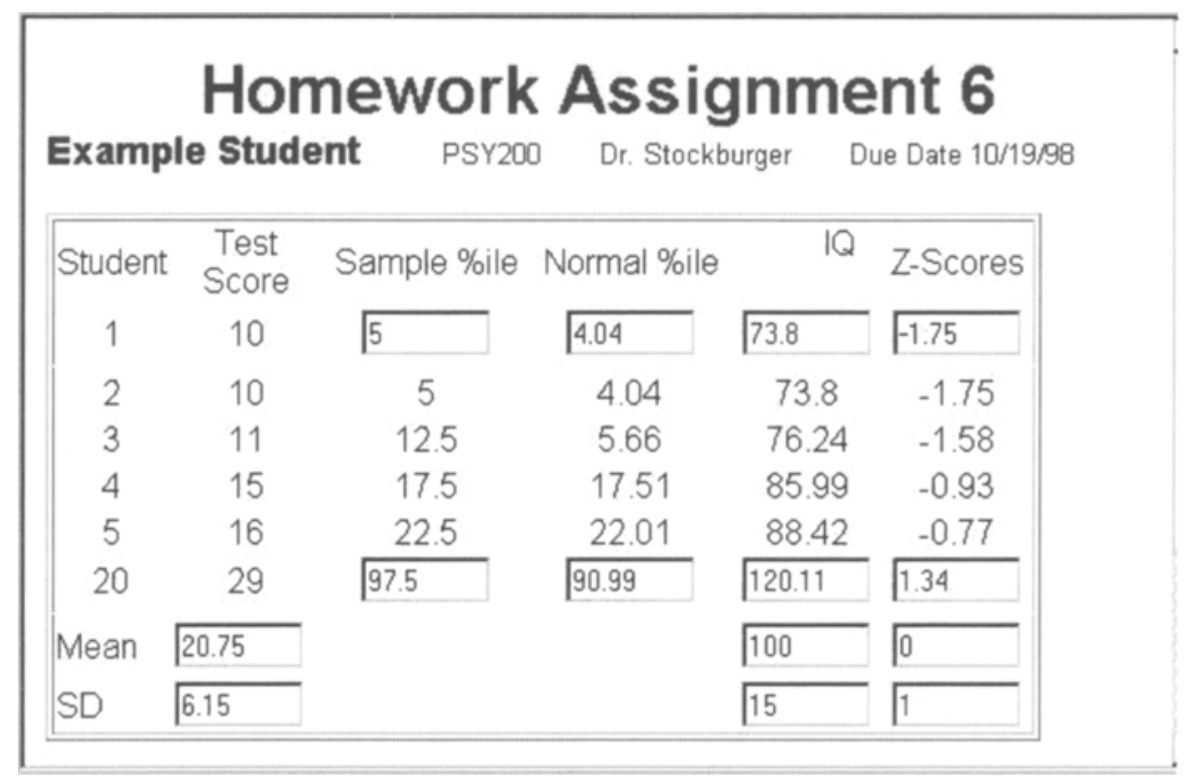

Figure 4. Example answer key.

The absence of variable typing is a common cause of difficulties when programming in either VBScript or JScript. For example, if $x 1=2$ and $x 2=5$, the sum, $x 1+$ $x 2$, will be 25 if $x 1$ and $x 2$ are text and 7 if they are integers. In order to ensure that the text box entries are numbers in the server-side script, the isNumeric() and $\mathrm{CDbl}()$ commands are used in combination. The parseFloat() command serves the same function in the client-side script.

Appearing throughout the ASP script are " $<\%="$ and "\%" combinations. When the page is sent to the user, the value of the variable appearing between these symbols will appear as part of the HTML code. This can be verified by examining the source code of the page that is sent to the user.

\section{Database Connections}

In combination with a basic understanding of ASP programming, database connections provide the Web programmer with powerful tools. A second example program demonstrates how an ASP program can access and display a simple database. The program listing is given as Listing 2, and an example HTML page is presented in Figure 6.

Setting up the program. The program and example Access database are included in the http://www. psychstat.smsu.edu/BRMIC/BRMIC.zip file. The program must be located in a folder in the same manner as the first example. In addition, to allow the program to access the database, an ODBC connection must be established. This connection can be done by using the following sequence of responses: Start, Settings, Control Panel, ODBC, System DSN, Add . .., Microsoft Access Driver,
Finish, Select, (locate the BRMIC database on the server), OK, enter a Data Source Name (the ASP program expects "BRMIC"), enter a Data Source Description, OK. An additional benefit from establishing this connection is that statistical packages that allow ODBC connections can directly access the database to set up data files.

Running the program. The user accesses the program like any other Web page, either by entering the URL in the browser or as a link from another page. Entering http://www.psychstat.smsu.edu/scripts/BRMIC/BRMI2.asp will run the example page from a remote location. As in the previous example, the page is recursively called when the user clicks on the "Submit" button. In this case, the server-side script checks to see if an "account" in the database matches the entry in the "Account Code" text box on the form. If a match is found, the full entry is displayed in the first section of the output. The reader is encouraged to change the database and view the results.

Program details. In addition to the details of the first example, this example establishes a connection with a database, selects records from the database, and sequentially displays the selected records. The connection with the database is done with the set dbBRMIC = Server. CreateObject("ADODB.Connection") command, where dbBRMIC could be any legal variable name. The dbBRMIC.Open "BRMIC" command tells the server the name of the database to open. This name must match a Datasource Name given when the ODBC connection was originally established.

A set of records (recordset) is selected from the database by executing an sql command. The details of sql commands are beyond the scope of this paper. In the exam- 
LISTING 1

Client and Service Side Programming Example

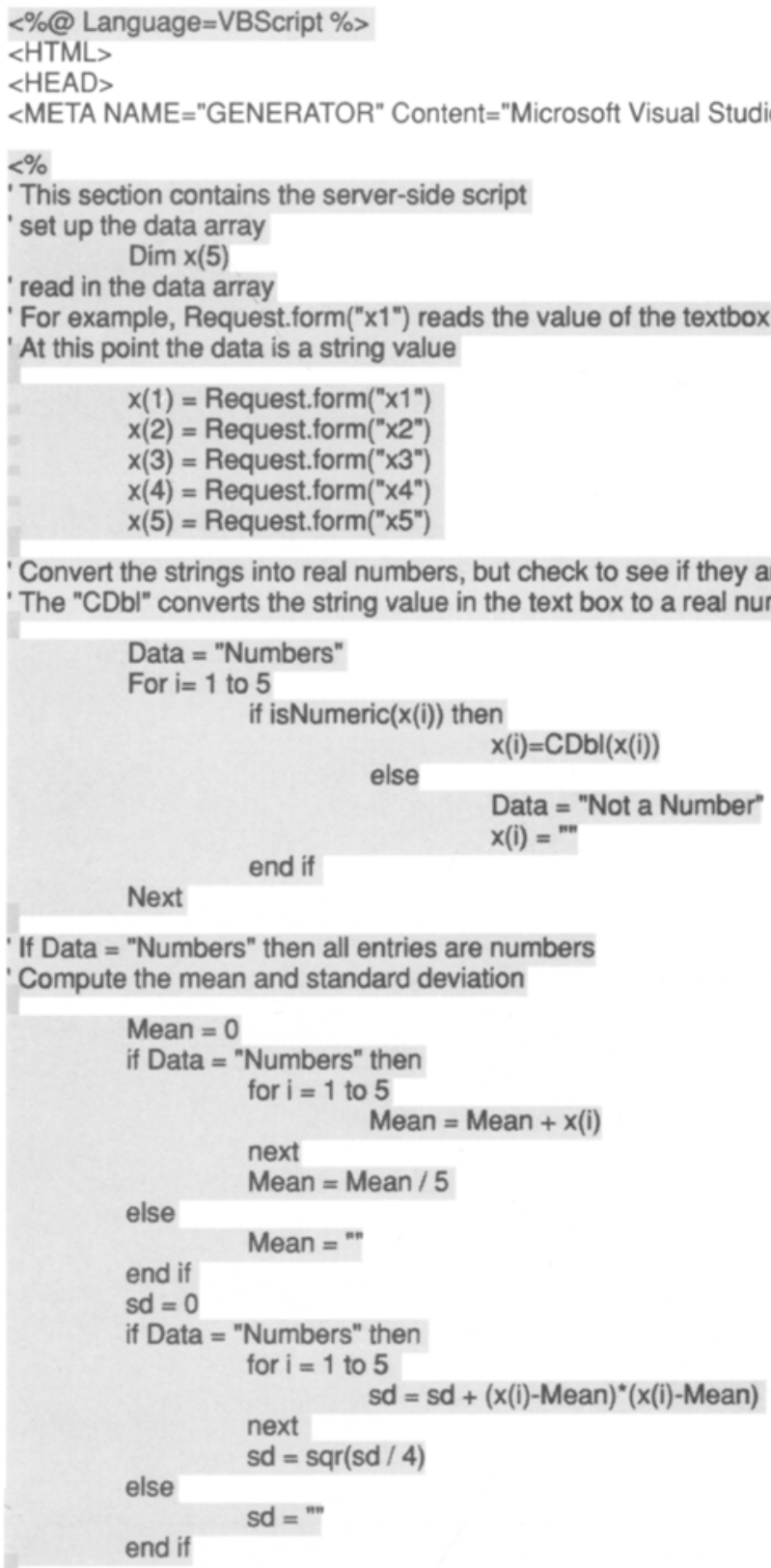

The Mean and sd variables are now ready to be used in the program

$\%>$

$<$ SCRIPT ID=clientEventHandlersJS LANGUAGE=javascript>

$<!--$

// This is a client script that is called when the ClientButton is clicked

function FindStats() \{

// set up the data array

$\operatorname{var} \mathrm{x}=$ Array $(5)$;

// read the values from the form into the data array

// for example " $\mathrm{x} 1$ " is the name of the textbox on the form named "data" in the html code below

// data.x1.value reads the current value of the text box " 1 1" on form "data"

// parseFloat(data. $x 1$.value) converts the string value to a floating point value

$x[1]=$ parseFloat(data.x1.value);

$x[2]=$ parseFloat(data. $x 2$.value);

$x[3]=$ parseFloat(data.$x 3 \cdot$ value); 
LISTING 1 (Continued)

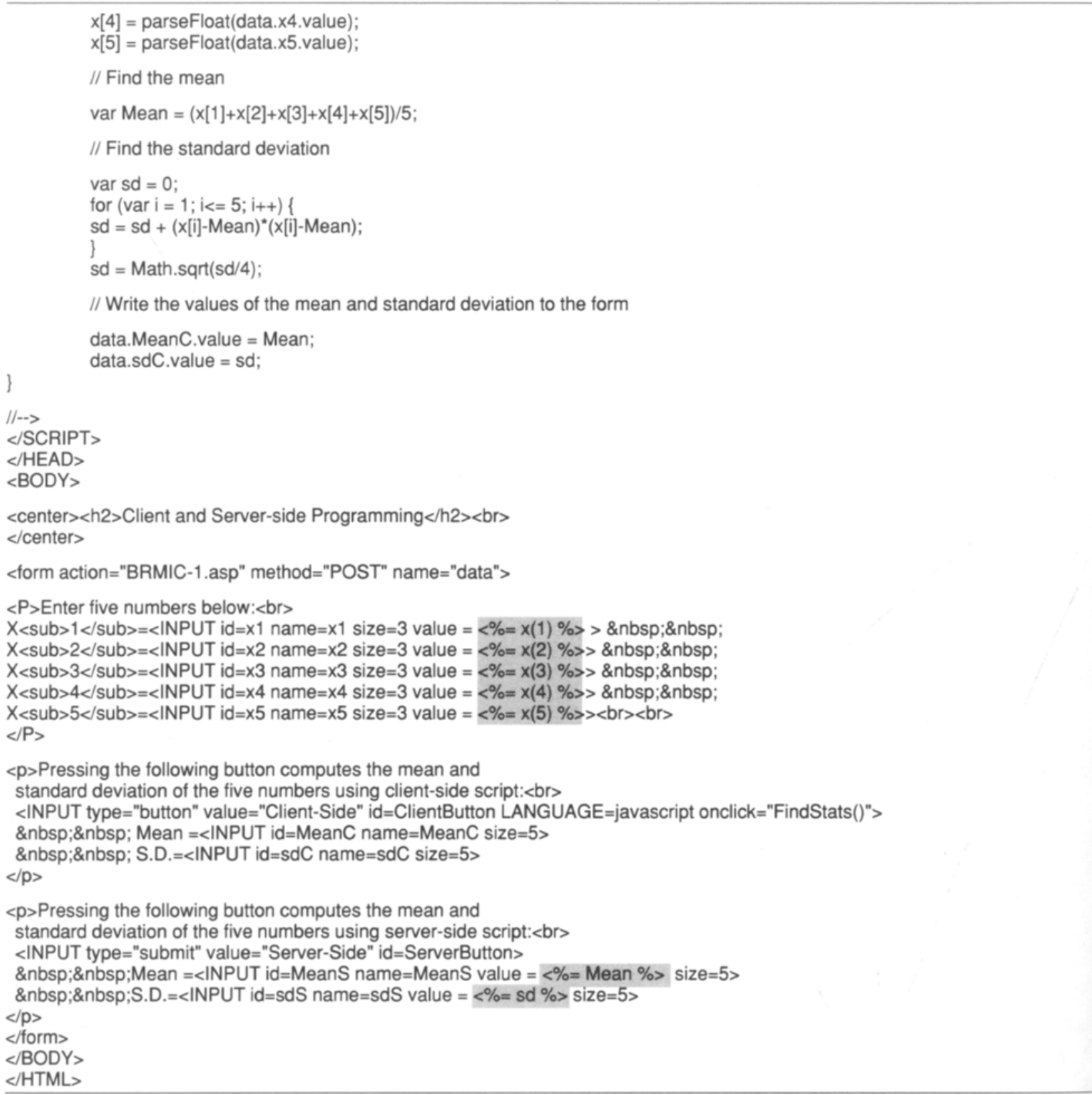

ple program, two recordsets are created. The first, named rsBRMIC, selects only database entries where the "Account Code" entered by the user on the form matches the "Account" entry in the database. The second, rsBRMICa, selects all entries in the database. The combination of commands sql = "some sql command" and Set rsBR$\mathrm{MIC}=$ dbBRMIC. Execute(sql) creates the recordset.

Looping through the recordset is done by the combination of Do While rsBRMIC.EOF, rsBRMIC.MoveNext, and Loop commands. At any point in the loop, data entries can be found by the rsBRMIC("variable") command, where "variable" is the name of a database field name.

\section{TECHNICAL ISSUES}

\section{Security}

Care has been taken to ensure that students cannot subvert the system. Security on the current system requires that students can submit each assignment only once and must not be able to observe the answer key before submitting the form. The main security system employed is to always assign a value other than zero in the database for the assignment after the student submits the assignment. Before grading the assignment, the program always checks to make sure the current grade is a zero. If 


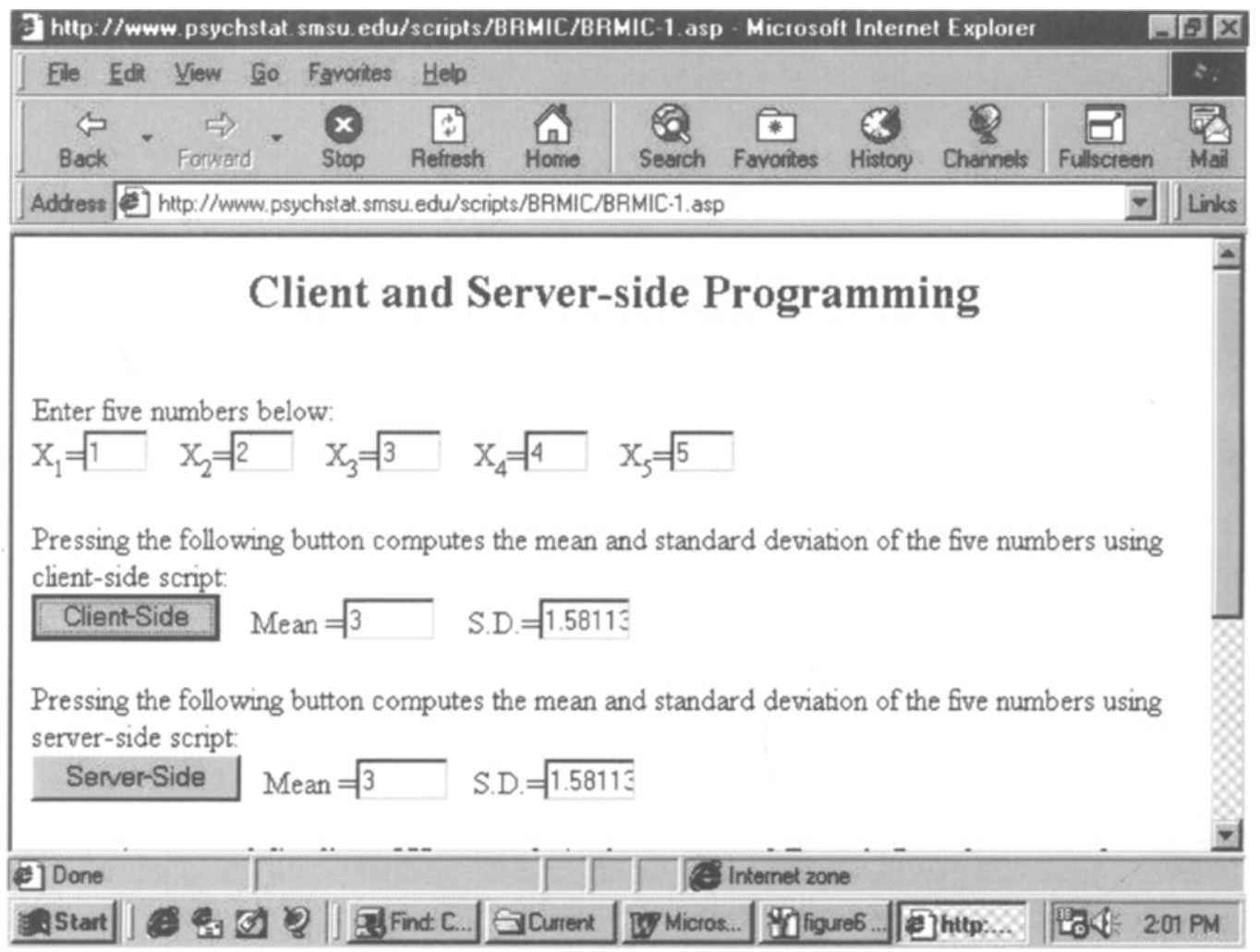

Figure 5. Output for client- and server-side programming example.

the assignment score for the student has some value other than zero, the program refuses to grade the assignment.

Storing all records on a database on the Web server requires security precautions against hacking into the system. A basic understanding of file and folder security on Windows NT is necessary, to allow instructors access to the database from computers either on or off campus, but to not allow others similar access.

\section{Availability}

A second technical issue is the availability of a server that can handle ASPs. At the present time, only Windowsbased servers have this capability, although that will probably change in the near future. The system administrators at many universities may be reluctant to allow script processing and database applications on the system that is hosting the university's Web pages. A possible response is to use a personal Web server on an office machine for ASP Web applications.

The Web servers included with Windows NT Workstation, Windows 98 , and various software packages such as Microsoft Frontpage are limited in capability. They work fine for development purposes and can handle most classroom sizes with little difficulty. If the popularity of a website increases, however, these systems will most likely be unable to handle the number of hits the system receives. Users who receive the "too many users" message might never return. One solution is a separate computer running Windows NT Server. It was found from experience with such a system that it could be set up in an afternoon, running without problems $24 \mathrm{~h}$ a day, 7 days a week, for over a year.

\section{Speed}

Generally speaking, scripts that run on the client are faster than scripts than run on the server. Server-side scripts require both a greater number of transfers between client and server and a greater number of processing cycles on the server. Twenty students simultaneously accessing the server, requesting their gradebook, tests, or assignments, does not seem to appreciably slow down the server.

\section{Compatibility}

The greater the demands on the client browser, the less likely that the client browser will be able to meet all the demands. A program that runs on a minimal system is said to be designed for a "thin" client. For example, if the programmer uses VBScript as the client-side scripting language, only users running Internet Explorer 3.0 or greater 
will be able to run the script. This is acceptable when the programmer has a certain amount of control over the computing environment, as would be the case if the assignments were to be run only in a given computer lab. If the programmer wishes to reach a larger, more diverse audience, compatibility issues must be considered.

Another issue is the use of the latest technology in designing Web pages. If the technology has stabilized, it seems reasonable to use the technology for courseware, even if some students find it necessary to upgrade their systems. The new technology must appreciably improve the product, however.

\section{OTHER APPLICATIONS OF THE TECHNOLOGY}

Connecting to a database from ASPs opens up a world of opportunities for Web programming. For example, 15 years of student evaluations of faculty results have been placed on the Web. Psychology faculty can access

LISTING 2

Database Programming Example

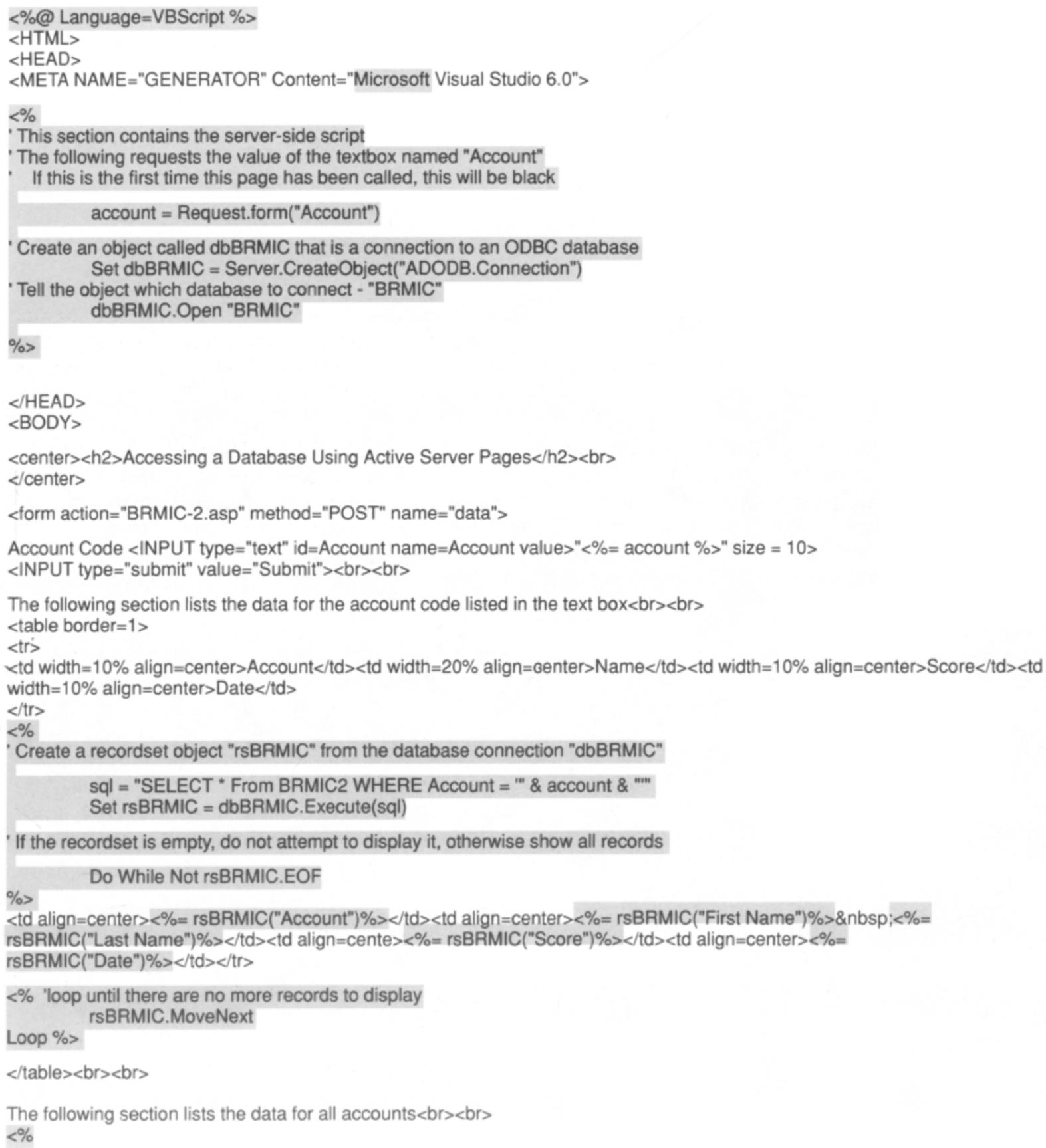




\section{LISTING 2 (Continued)}

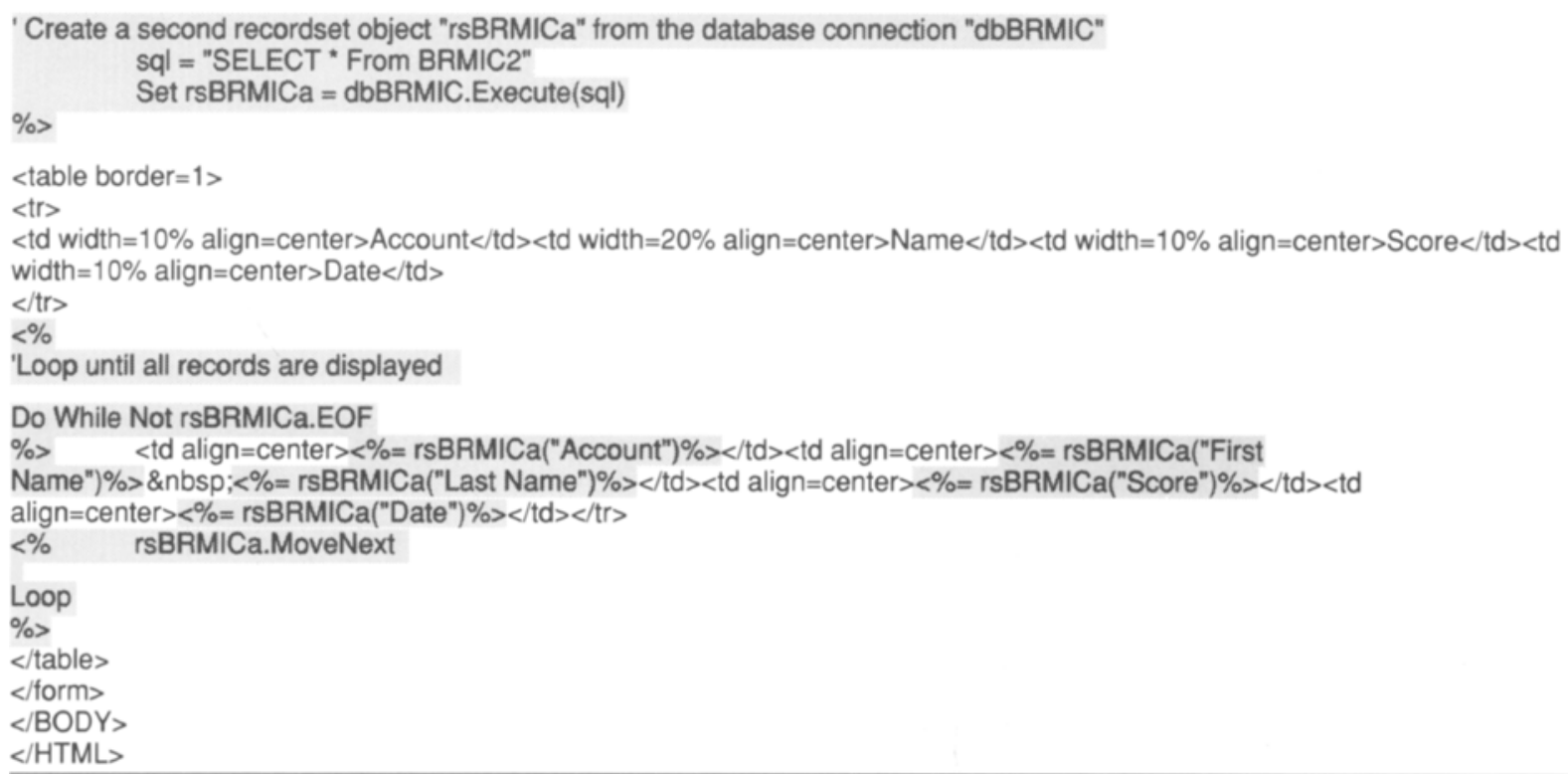

the results for any course they taught at any time during that period. They can norm the results of a particular class against the whole faculty, the same course taught by other instructors, or only their own sections of that course.

Another application is a listing of Web resources. It is much easier to update and manipulate a database than to edit HTML pages. An example of this application can be seen by selecting the "Statistical Resources without Pictures" [at http://www.psychstat.smsu.edu].

Applicants for faculty positions in the psychology department in our university can retrieve information about the completeness of their application file at any time on

E hilp://www. psychstat. smsu edu/scripts/BRMIC/BRMIC-2.asp-Microsoft Internet Explorei $\quad$ -

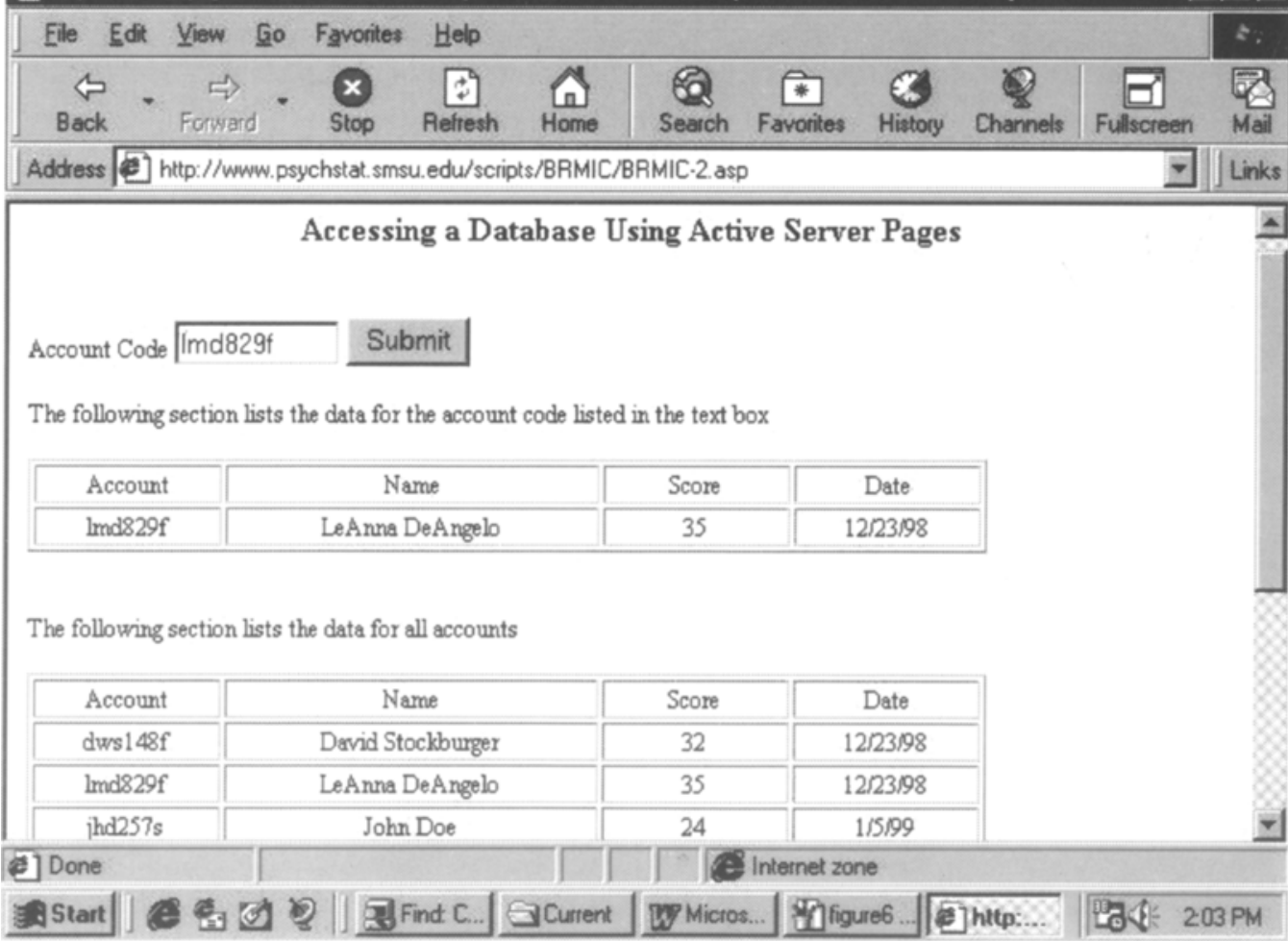

Figure 6. Output for database programming example. 
a personalized Web page. Limited changes in phone and address information are possible in their own database record. Similar utility programs are planned for graduate school applicants. Vast arrays of potential applications are available for a minimal time investment in learning about ASP.

\section{REFERENCES}

Corning, M., Elfanbaum, S., \& Melnick, D. (1997). Working with active server pages. Indianapolis: QUE.

Kenton, C. (1998). Following the function of forms. WEB Builder, 3 , 69-72.

STOCKBURGER, D. W. (1996, October). Generating individualized tests and homework assignments using HTML as a prototype form. Paper presented at the annual meeting of the Society for Computers in Psychology, Chicago.

\section{APPENDIX \\ Web Resources}

Zipped file of example programs:

http://www.psychstat.smsu.edu/brmic/brmic.zip

Client and Server-side Programming Example:

http://www.psychstat.smsu.edu/scripts/brmic/brmic-1.asp

Database Programming Example:

http://www.psychstat.smsu.edu/scripts/brmic/brmic-2.asp

Access to course pages, lists of statistical resources, and on-line texts:

http://www.psychstat.smsu.edu

(Manuscript received October 19, 1998;

revision accepted for publication January 8,1999 .) 